\title{
Evaluation of the Efficiency of Solvent Systems to Remove Acetic Acid Derived from Pre-pulping Extraction*1
}

\author{
Seong-Jik Park*2, Joon-Kwan Moon*3, and Byung-Hwan Um*4†
}

\begin{abstract}
Hemicellulose extract obtained by pre-pulping extraction of woodchips, is very diluted acidic in nature. The major component responsible for this weak acidity is acetic acid, present in levels up to $5 \sim 10 \mathrm{~g} / \mathrm{L}$. Here, we report an exploratory study on the extract upgrading by reactive solvent extraction of acetic acid as well as ASPEN simulation. In this study, liquid-liquid equilibria for the ternary systems (water + acetic acid + ethyl acetate) were measured at the temperature of $298.15 \mathrm{~K}$ and $10(\mathrm{pH}=2.02), 5(\mathrm{pH}=2.17)$, and $1(\mathrm{pH}=2.48)$ percent of acetic acid samples were used to carry out liquid-liquid extraction studies using ethyl acetate. In a one-stage batch experiment, $96.0 \%$ of acetic acid could be extracted for the solvent when the ratio of organic-to-aqueous phases is $4: 1$. For simulation results, they were used to estimate the interaction parameters between each of the three compounds of the systems studied for the NRTL and UNIQUAC models. The estimated interaction parameters were successfully used to predict the equilibrium compositions by the two models.
\end{abstract}

Keywords : acetic acid, ASPEN, liquid-liquid extraction (LLE), pre-pulping extract, simulation

\section{INTRODUCTION}

The pulp mill-based industry can be utilized for the production of biofuels and chemical products through the pre-pulping extraction of hemicelluloses. In the hemicellulose extract conversion, the extract contains mostly water, hemicellulose, and acetic acid before it is fermented to create bioethanol. Some decomposed com- pounds, mainly acetic acid, hinder the subsequent bioconversion of the solubilized sugars into desired products by reducing fermentation rates and conversion yields (Um et al., 2011). The pulp and biorefinery is conceptualized as a collection of the various process options described above (Fig. 1).

Acetic acid is one of the most widely used carboxylic acids. It is used in many reactions, for example, the synthesis of acetic ester or it

*1 Received on June 18, 2013; accepted on September 17, 2013

*2 Department of Bioresources and Rural System Engineering, Hankyong National University, Gyeonggi-do, 456-749, Korea

*3 Department of Plant Life and Environmental Science, Hankyong National University, Gyeonggi-do, 456-749, Korea

*4 Department of Chemical Engineering and Research Center of Chemical Technology, Hankyong National University, Gyeonggi-do, 456-749, Korea

† Corresponding author : Byung-Hwan Um (e-mail: bhum11@hknu.ac.kr) 


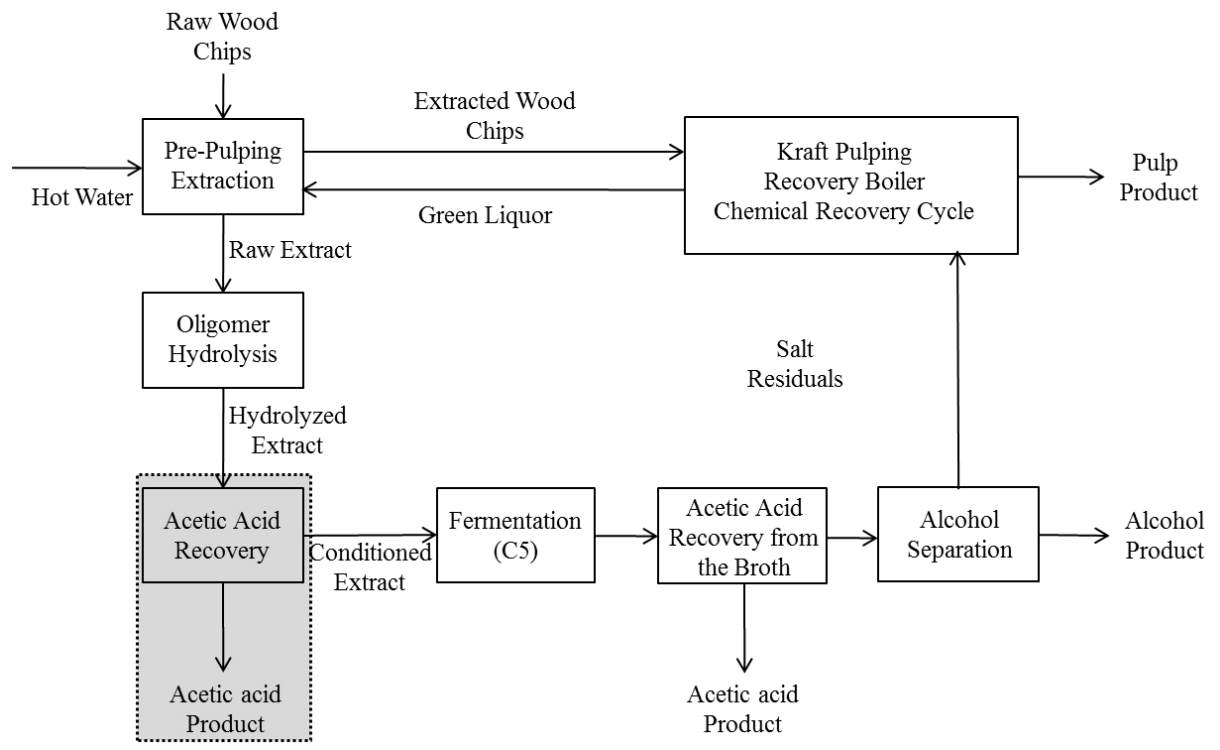

Fig. 1. BFD (block flow diagram) representing the process flow of a pulp and biorefinery. The Acetic acid recovery steps are the focus of this study.

can be used as a solvent. The separation of acetic acid from its aqueous solution by simple rectification is very difficult, requiring a column with many stages and a high reflux ratio, thus incurring high running costs (Wasewar et al., 2004; Anasthas et al., 2001). In practice other processes are also used depending on the concentration of acetic acid present in the feed. Between 50\% and 70\% w/w acetic acid, extractive distillation is used. By adding a third component, the volatility of water is increased and the separation can be achieved with less energy (Smejkal et al., 2005). Below 40\% acetic acid, liquid-liquid extraction with single solvent is most appropriate. Acetic acid is extracted from water by a suitable solvent in order to obtain substantially pure acetic acid (Renon and Prausnitz., 1968). The BFD (block flow diagram) in Fig. 1 shows the pulp and biorefinery concept of acetic acid recovery.

The recovery of acetic acids from the extract solutions prior to fermentation processes is in- dustrially important, and many solvents have been tried to improve such recovery by means of liquid-liquid extraction. In separation and purification technology the liquid-liquid extraction plays an important role. The liquid-liquid extraction is used to separate two miscible liquids using a solvent that preferentially dissolves one of them (McCabe et al., 1993; Arce et al., 1995; Braones et al., 1994; Dramur and Tatli., 1993). The three branches of solvent extraction are the traditional applied field, the nuclear material field and the theoretical field (Ritcey and Ashbrook., 1979). This is a low temperature and a low cost process. Metals are also purified by this process. Close boiling mixtures or substances can also be separated by this method (Marian et al., 2006). The equilibrium relationship of the components involved is of prime importance in solvent extraction. The equilibrium relationship in liquid extraction is generally more complicated than for other separations, because there are three or more compo- 
nents present in each phase. So, the equilibrium relationships are presented on a triangular diagram (Marian et al., 2006). Precise liquid-liquid equilibrium data are required for extraction processes. Excess activity models, such as the nonrandom two-liquid model (NRTL) (Renon and Prausnitz., 1968; Dramur and Tatli., 1993) and the universal quasi-chemical theory (UNIQUAC) (Abrams and Prausnitz., 1975; Arce et al., 1995), have been successfully applied for the prediction of several liquid-liquid systems. In each case, the adjustable parameters were obtained by regressing the experimental data to the models and obtaining numerical values for the interaction parameters. The NRTL and UNIQUAC models depend on experimentally optimized interaction parameters between each two molecules in the system.

The studies listed above were focused on removal of acetic acids and did not investigate the suitability of the extracted aqueous phase for fermentation. Application of ethyl acetate extraction to pre-pulping hemicelluloses extract is a new application of the technology and there are few data in the published literature that indicate how extraction of acetic acid will alter the fermentable sugar levels. The objective of this work was to study the liquid-liquid phase equilibria of the ternary systems water + acetic acid + ethyl acetate at $298.15 \mathrm{~K}$ and $1 \mathrm{~atm}$. The present study also evaluates the extraction yield of acetic acid and mass balance closure for the solvent extraction as three different acid concentrations.

\section{MATERIALS AND METHODS}

\subsection{Experiments}

A synthetic extract was prepared in the laboratory from standard chemicals and deionized water; a mixed solution of $10 \mathrm{~g} / \mathrm{L}$ acetic acid
(Sigma Aldrich, $>99 \%$ purity, St. Luis, MO) was used as a model pre-pulping extract. The $\mathrm{pH}$ of the solution was measured to be at 2.48. The ethyl acetate solvent was run at five different solvent-to-feed ratios of $0.2,0.5,1.0,2.0$, and 4.0 for liquid-liquid extraction (LLE) of acetic acid. A typical extraction procedure for each experiment was initiated by the addition of the organic phase and aqueous solution into a $250 \mathrm{ml}$ separatory funnels. It was conducted in a batch mode with vigorously shaking for 6 seconds, which was shaken in every six minutes at $298.5 \mathrm{~K}$ and $1 \mathrm{~atm}$. The sample was then settled down at $298.5 \mathrm{~K}$ for 20 minutes to allow separation of the two phases. Samples of both phases were analyzed immediately after the phase separation.

\subsection{Analysis}

The concentration of acetic acid in the aqueous phase (bottom) were determined by Shimazu HPLC (high performance liquid chromatography) with RI (refractive index) detector and Bio-Rad Aminex HPX-87H column (Bio-Rad Laboratories Inc., Hercules, CA) and HPLC column operation conditions were column temperature of $65^{\circ} \mathrm{C}$, flow rate of $0.6 \mathrm{~m} \ell / \mathrm{min}$, mobile phase of $0.005 \mathrm{M}$ sulfuric acid. The concentration of acetic acid in the organic phase (top) was determined by GC (gas chromatography, Shimadzu model GC-2010, Shimadzu Corp., Kyoto, Japan), equipped with a FID (flame ionization detector) and using a Rtx 5 column (Restek Corp., Bellefonete, PA). The GC injector and detector temperatures were $280^{\circ} \mathrm{C}$ and $340^{\circ} \mathrm{C}$, respectively. The carrier gas was $\mathrm{He}$ at $30 \mathrm{ml} / \mathrm{min}$.

\subsection{Partition Coefficient $\left(P_{c}\right)$ and Extraction Yield (\%)}

In order to characterize the equilibrium parti- 


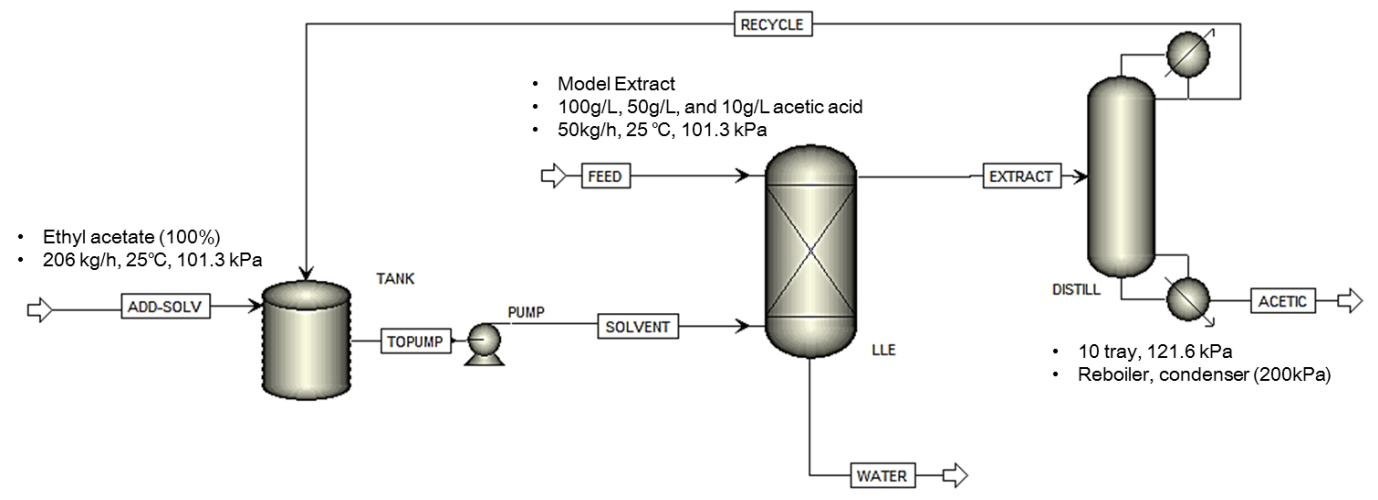

Fig. 2. PFD (process flow diagram) simplified by ASPEN representing the process of aceticacid extraction by ethyl acetate.

tioning of acetic acid between the two phases, the partition coefficient $\left(\mathrm{P}_{\mathrm{c}}\right)$ was calculated at the different tested conditions. The $\mathrm{P}_{\mathrm{c}}$ is defined as:

$$
P_{c}=\left[\frac{[H A c]_{\text {org }}}{[H A c]_{a q}}\right]
$$

where [HAc] is acetic acid concentration; subscript "org" is the designation of the acid concentration extracted from the aqueous phase in the organic phase; and subscript "aq" is the designation of the acid concentration remaining in the aqueous phase. An alternate means of expressing extraction efficiency is percent extraction. Percent extraction is defined as the fraction of acetic acid removed from the aqueous phase into the organic phase in one extraction step, as shown in equation below:

$$
\% \text { Extraction }=\left(1-\frac{[H A c]_{\text {remaining } a q}}{[H A c]_{\text {initial aq }}}\right) \times 100
$$

\subsection{ASPEN Simulation}

ASPEN HYSYS software (Version 7.1) was used to simulate the liquid-liquid extraction of acetic acid from a model mixture of extracts, i.e., 10, 5, and 1 percent of acetic acid using ethyl acetate as a solvent. If a liquid mixture of a given composition and at known temperature is separated into two phases (at equilibrium), the compositions of the two phases can be calculated using the following system of equations.

$$
\gamma_{i}^{E} x_{i}^{E}=\gamma_{i}^{R} x_{i}^{R}
$$

Where $x_{i}^{E}$ and $x_{i}^{R}$ are the moles fraction of component $i$ in the extract (organic) phase and in the raffinate (aqueous) phase, respectively, and $\gamma_{i}^{E}$ and $\gamma_{i}^{R}$ are the corresponding activity coefficients of component $i$ in the extract and raffinate phases, as calculated from the equilibrium model, i.e., NRTL or UNIQUAC. The interaction parameters between water, acetic acid, and ethyl acetate are used to estimate the activity coefficients from NRTL and UNIQUAC equations. The counter-current liquid-liquid extraction simulation with solvent distillation was simulated to observe both the effectiveness of ethyl acetate as a solvent in a counter current liquid-liquid extraction procedure and the recyclability of ethyl acetate. Fig. 2 shows the ASPEN layout of the simulation. 
Evaluation of the Efficiency of Solvent Systems to Remove Acetic Acid Derived from Pre-pulping Extraction

Table 1. Equilibrium data for extraction of acetic acid from model solution as function of different ratio org: aq with ethyl acetate

\begin{tabular}{ccccc}
\hline \multirow{2}{*}{$\begin{array}{c}\text { Ratio of org:aq } \\
{[\mathrm{mL} / \mathrm{mL}]}\end{array}$} & \multicolumn{2}{c}{$\begin{array}{c}\text { Acetic acid concentration } \\
{[\mathrm{g} / \mathrm{L}]}\end{array}$} & $\begin{array}{c}\text { Partition coefficient, } \\
\text { [org/aq] }\end{array}$ & $\begin{array}{c}\text { Extraction } \\
{[\%]}\end{array}$ \\
\cline { 2 - 5 } & $\mathrm{aq}^{\mathrm{a}}$ & $\mathrm{org}^{\mathrm{b}}$ & $\mathrm{P}_{\mathrm{c}}$ & 59 \\
\hline \hline $1.00[10: 10]$ & 4.1 & 5.9 & 1.4 & 74 \\
$1.50[15: 10]$ & 2.6 & 4.9 & 1.9 & 82 \\
$2.00[20: 10]$ & 1.8 & 4.1 & 2.3 & 88 \\
$2.50[25: 10]$ & 1.2 & 3.5 & 2.9 & 93 \\
$3.00[30: 10]$ & 0.7 & 3.1 & 4.4 & 97 \\
$4.00[40: 10]$ & 0.3 & 2.4 & 8.1 & \\
\hline
\end{tabular}

Note: acetic acid measured by calculation ${ }^{\mathrm{a}}$ and $\mathrm{GC}^{\mathrm{b}}$

The experimental conditions: Feed concentration $=10 \mathrm{~g} / \mathrm{L}, \mathrm{pH}=2.48$, temperature $=298.15 \mathrm{~K}$, and contact time $=36$ minutes

\section{RESULTS AND DISCUSSION}

For the feed containing 10, 5, and 1 percent of acetic acid, the separation from aqueous solvent with ethyl acetate has been compared with simulated partition coefficient values and partition coefficient obtained in the present experimental work. The laboratory based simulations that consisted of only LLE showed that the acetic acid could be effectively removed from the feed mixture using ethyl acetate. Analysis of the experimental distribution coefficients obtained for various feed concentrations and different solvent ratio in the Table 1. With initial feed concentrations of $10 \mathrm{~g} / \mathrm{L}$ by weight of acetic acid in water, the amount of acetic acid recovered was $96 \%$ at the ratio of 4 (Table 1). From the laboratory experiments, it shows that for feed containing lower concentrations of acetic acid, ethyl acetate alone is suitable. It is also observed from the $P_{c}$ values that for the feed containing higher concentrations of ethyl acetate are not able to give any result and this due to the co-extract, the water from the solvent (Abrams and Prausnitz., 1975). Whereas for the feed containing higher concentration of acetic acid, increase in the value of partition coefficient in this case is due to the favored conditions arising from the combined action. The increase in partition coefficient for the case of solvent extraction is being explained using ternary diagram drawn in Fig. 3.

\subsection{Ternary Equilibrium Diagram for LLE Process}

From the equilibrium diagram for the ternary systems ethyl acetate / acetic acid / water was simulated. And shown in Fig. 3, the two phase region is quite small for ethyl acetate / acetic acid / water shown in Fig. 3a, limiting the maximum feed concentration for the liquid to liquid extraction. However, the two phase region for the ternary system ethyl acetate / acetic acid / water is large (Fig. 3b) and therefore can be used for higher feed concentration. Similar bimodal curve for the ternary system of acetic acid/water/ethyl acetate have been drawn using 

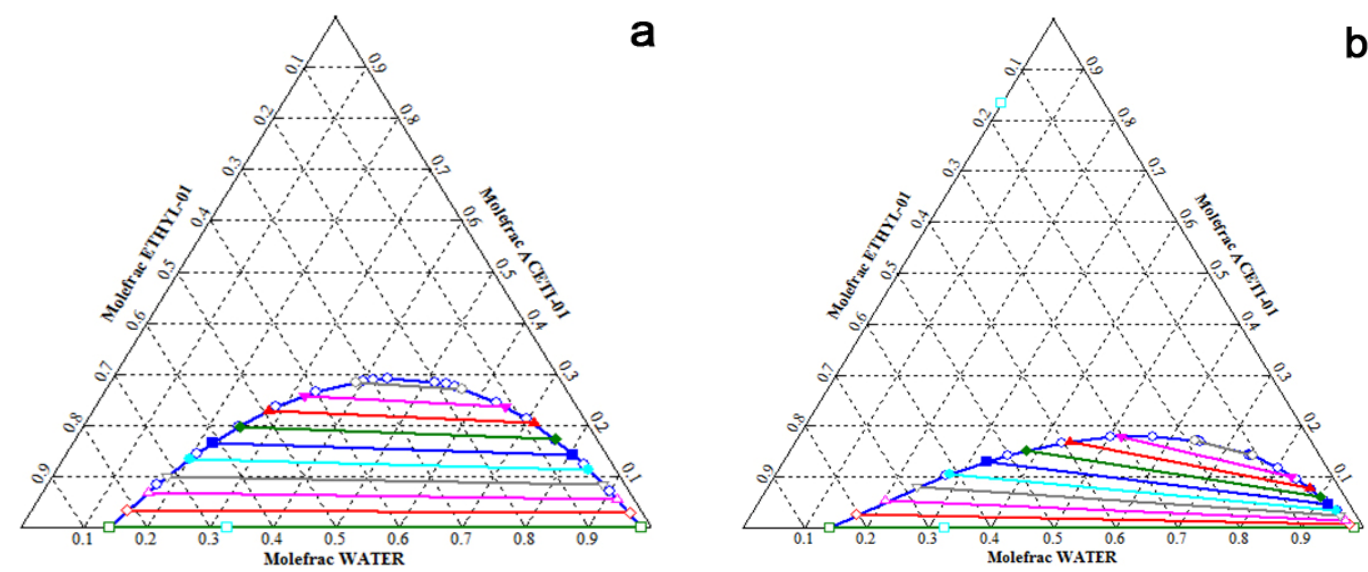

Fig. 3. Ternary map for water, acetic acid and ethyl acetate mixture: (a) $\mathrm{T}=298.3 \mathrm{~K}$, model NRTL; (b) $\mathrm{T}=298.3$ $\mathrm{K}$, model UNIQUAC.
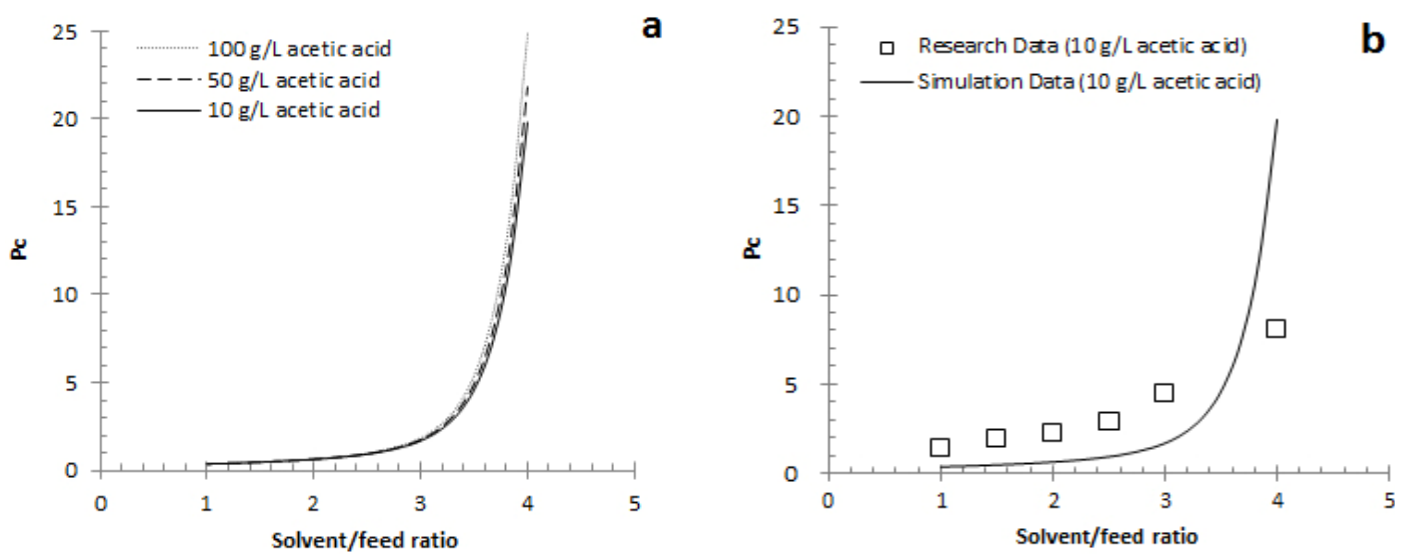

Fig. 4. Partition coefficient $\left(\mathrm{P}_{\mathrm{c}}\right)$ profiles determined from (a) ASPEN HYSYS simulation and (b) comparison of partition coefficients $\left(\mathrm{P}_{\mathrm{c}}\right)$ for research and simulation data.

the calculated tie line data from the present experimental work. The models NRTL and UNIQUAC were successfully used to regress the experimental equilibrium compositions of both studied systems.

\subsection{Model Predictions and Comparison of Partition Coefficient}

NRTL and UNIQUAC models in ASPEN simulation software were used to find the partition coefficients $(\mathrm{Pc})$ for the feed concentration of 10, 5, and 1 percent of acetic acid (Fig. 4a). The calculated Pc values were compared with experimental results in Fig. 4b. This shows that UNIQUAC can predict the results which are in good comparison with the experiment results. Table 1 shows the experimental yield for extraction of acetic acid from aqueous synthetic extract at $25^{\circ} \mathrm{C}$ as a function of the solvent- 
to-feed ratio. The relative concentration of acetic acid that was extracted into the organic phase decreased from $5.9 \mathrm{~g} / \mathrm{L}$, corresponding to $69.0 \%$ extraction yield, at a ratio of 1 to a concentration of $2.4 \mathrm{~g} / \mathrm{L}$, i.e. $96.0 \%$ extraction yield, at a ratio of 4.0. An increase in the solvent-to-feed ratio from 1 to 4 produced a linear increase of $\mathrm{P}_{c}$ from 1.4 to 8.1.

Comparing the results derived from simulation and experiment according to the mass fraction obtained in the extract of acetic acid, the maximum partition coefficients were 19.8 and 8.1 respectively (Fig. 4b). Given that the laboratory results were obtained using batch small scale equipment, a difference of 11.7 is substantial at the ratio of $4: 1$. The large discrepancy between the $P_{c}$ of the continuous counter-current and batch LLE processes shown in Fig. $4 \mathrm{~b}$ indicates that future research should thoroughly evaluate the feasibility of LLE in an industrial setting given that the counter-current extraction is currently believed to be the most apposite method for separation. However, the simulations were a mathematical prediction of what would happen if the ideal circumstances had occurred in the plant. The main factor causing deviations from the simulations was the design of the column in the continuous LLE. The simulated column was comprised of a packed bed where in reality it is possible to predict the exact outcome due to the laminar counter-current mixing that occurs.

\subsection{Recyclability of Solvent for LLE Process}

To simulate the recycling of the ethyl acetate in the distillate back into the solvent stream of the LLE, an entirely separate LLE and distillation column was added in series beside the existing simulation, as shown in Table 2. The distillate stream containing approximately 94\%
Table 2. The effect of distillation rate on acetic acid flow rate and purity in the product

\begin{tabular}{|c|c|c|}
\hline $\begin{array}{l}\text { Distillate } \\
\text { rate }(\mathrm{kg} / \mathrm{s})\end{array}$ & $\begin{array}{l}\text { Acetic acid } \\
\text { Rate }(\mathrm{kg} / \mathrm{s})\end{array}$ & $\begin{array}{l}\text { Acetic acid Purity } \\
\text { (Mass fraction) }\end{array}$ \\
\hline 3.0000 & 0.0986 & 0.0844 \\
\hline 3.0605 & 0.0986 & 0.0890 \\
\hline 3.1210 & 0.0986 & 0.0941 \\
\hline 3.1815 & 0.0986 & 0.0999 \\
\hline 3.2421 & 0.0985 & 0.1064 \\
\hline 3.3026 & 0.0985 & 0.1139 \\
\hline 3.3631 & 0.0985 & 0.1224 \\
\hline 3.4236 & 0.0985 & 0.1323 \\
\hline 3.4842 & 0.0985 & 0.1440 \\
\hline 3.5447 & 0.0985 & 0.1580 \\
\hline 3.6052 & 0.0985 & 0.1750 \\
\hline 3.6657 & 0.0985 & 0.1961 \\
\hline 3.7263 & 0.0984 & 0.2229 \\
\hline 3.7868 & 0.0984 & 0.2583 \\
\hline 3.8473 & 0.0984 & 0.3069 \\
\hline 3.9078 & 0.0984 & 0.3783 \\
\hline 3.9684 & 0.0984 & 0.4928 \\
\hline 4.0000 & 0.0983 & 0.5853 \\
\hline 4.0289 & 0.0983 & 0.7066 \\
\hline 4.0894 & 0.0780 & 0.9932 \\
\hline 4.1500 & 0.0180 & 0.9965 \\
\hline
\end{tabular}

ethyl acetate was inserted into the bottom inlet of the LLE as the solvent. Extract stream was the distillate stream that was recycled and used as the solvent steam in the LLE column. The results of the first recycle were streams distillate and bottoms. The feed stream for the new LLE column was defined from the previous feed stream because the properties of this stream 


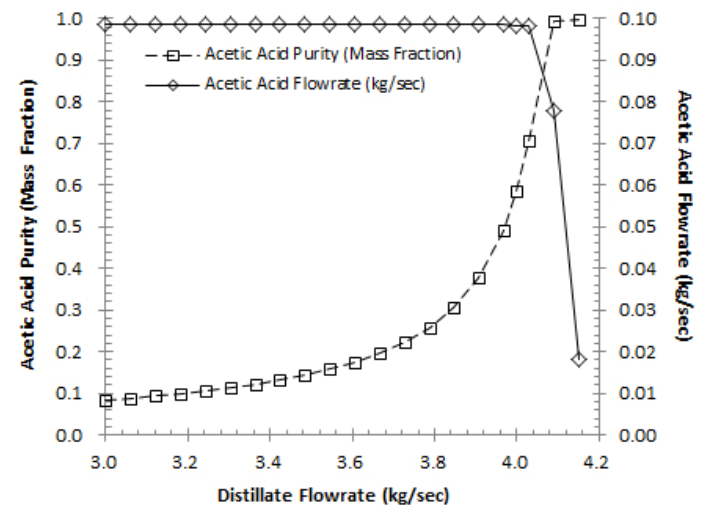

Fig. 5. Profile of distillation rate for recovery of acetic acid and purity in the product.

must not change. This process was repeated 24 times (data not shown); the same molecule of ethyl acetate ran through the process 24 times. A total of 25 LLE columns and distillation towers were designed in order to simulate 24 recycles. For recovering solvent and acetic acid, the parameter of distillation column was investigated. In the distillation column these are reflux ratio, feed position and distillate rate. Normally, feed goes in the middle of column and because of high relative volatile of ethyl acetate to acetic so reflux ratio is low (above minimum reflux ratio). One reflux ratio is set for distillation column. Table 2 presents the effect of distillation rate for recovery of acetic acid. Through the simulation the distillation column should run for recovering of high purity acetic acid at around $4 \mathrm{~kg} / \mathrm{s}$ distillate rate (Fig. 5).

\section{CONCLUSIONS}

In this study, the effect of solvent on liquidliquid equilibrium has been analyzed in terms of partition coefficients and ternary diagram and the suitable models for estimating the partition coefficients discussed. The partition coefficients for various acetic acid feed concentrations were studied using different ratio of solvent to feed. The liquid-liquid equilibrium was drawn in the form of ternary graph for the feed containing 10,5 , and 1 percent of acetic acid. The partition coefficient data were obtained using models available in ASPEN software and the simulation showed that pure recovery of the acetic acid should happen if the solvent to feed mass ratio is high enough. Also, starting with a mass flow ratio of 4:1 showed that the solvent could be recycled 24 times before resulting in a mass flow ratio of $1: 1$ which would then need to be replenished. Though it could appreciate the effect of ethyl acetate solvent on improving the extraction efficiency, as an extension of this work, further investigations can be done because the effect of property change and temperature on mixing have not been taken into consideration. However, this study would definitely give a first hand knowledge about the problem and provide a good insight into the complex and conflicting of the parameters governing the process.

\section{ACKNOWDGEMENT}

This work was supported by the International Cooperation of the Korea Institute of Energy Technology Evaluation and Planning (KETEP) grant funded by the Korea Government Ministry of Trade, Industry \& Energy. The authors also thank the National Research Foundation of Korea (NRF) grant funded by the Korea government (MEST), Project No. 2012R1A1A1003107 for financial support.

\section{REFERENCES}

1. Abrams, D. S. and J. M. Prausnitz. 1975. Statistical thermodynamics of liquid mixtures: A new expression for the excess Gibbs energy of partlyor completely miscible systems. AIChE J 
Evaluation of the Efficiency of Solvent Systems to Remove Acetic Acid Derived from Pre-pulping Extraction

21: $116 \sim 128$.

2. Anasthas, H. M. and V. G. Gaikar. 2001. Removal of acetic acid impurities from ethyl acetate by adsorption on ion exchange resins. Sep. Sci. Technol 36: 2623 2646.

3. Arce, A., A. Blanco, P. Souza, and I. Vidal. 1995. Liquid-liquid equilibria of the ternary mixtures water + propanoic acid + methyl ethyl ketone and water + propanoic acid + methyl propyl ketone. J. Chem. Eng. Data 40: 225 229.

4. Briones, J. A., J. C. Mullins, and M. C. Thies. 1994. Liquid-liquid equilibria for the oleic acidâ-sististerol-water system at elevated temperatures and pressures. Ind. Eng. Chem. Res 33: $151 \sim 156$.

5. Dramur, U. and B. Tatli. 1993. Liquid-liquid equilibria of water + acetic acid + phthalic esters (dimethyl phthalate and diethyl phthalate) ternaries. J. Chem. Eng. Data 38: 23 25.

6. Marian, G., W. G. Barbara, and M. Andrzej. 2006. Recommended liquid-liquid equilibrium data. J. Phys. Chem 35: 1391 1414.
7. McCabe, W. L., Smith and J. C. Harriott. 1993. Unit operations of chemical engineering $5^{\text {th }}$ ed.

8. Renon, H. and J. M. Prausnitz. 1968. Local composition in thermodynamics excess functions for liquid mixtures. AIChE J 14: 135 144.

9. Ritcey, G. M. and A. W. Ashbrook. 1979. Solvent extraction. Elsevier scientific publishing company.

10. Smejkal, Q., D. Linke, and M. Baerns. 2005. Energetic and economic evaluation of the production of acetic acid via ethane oxidation. Chem. Eng. Process 44: 421 428.

11. Um, B. H., B. Friedman, and G. P. van Walsum. 2011. Conditioning hardwood-derived pre-pulping extracts for use in fermentation through removal and recovery of acetic acid using trioctyl phosphine oxide (TOPO). Holzforschung 65(1): $51 \sim 58$.

12. Wasewar, K. L., A. A. Yawalkar, J. A. Moulijn, and V. G. Pangarkar. 2004. Fermentation of glucose to lactic acid with reactive extraction, a review. Ind. Eng. Chem. Res 43: 5969 5982. 